\title{
AVALIAÇÃO DA RIGIDEZ DE VIGAS ESTRUTURAIS DE MADEIRA LAMINADA COLADA UNIDAS POR ADESIVO POLIURETANO
}

\author{
Alexsandro Bayestorff da Cunha ${ }^{1}$, Jorge Luis Monteiro de Matos ${ }^{2}$
}

(recebido: 6 de maio de 2010; aceito: 22 de agosto de 2011)

\begin{abstract}
RESUMO: Conduziu-se este estudo, com o objetivo de avaliar tecnicamente a produção de vigas de Madeira Laminada Colada unidas por meio de adesivo poliuretano, pela determinação do módulo de elasticidade estático e dinâmico. Para a confecção das vigas estruturais, foram utilizadas peças de madeira serrada de Pinus taeda e adesivo Poliuretano. Todas as peças que fizeram parte das vigas foram classificadas visualmente pelo número de anéis de crescimento e porcentagem de lenho tardio, além da presença de defeitos. Seguidamente, as lamelas foram classificadas pelo módulo de elasticidade dinâmico. As vigas foram montadas e prensadas, tendo o valor do módulo dinâmico estimado e o estático calculado com base no ensaio em uma máquina universal. A análise dos dados foi realizada pela análise da regressão. Concluiu-se que a classificação visual utilizada na seleção de peças foi insuficiente para se atingir os valores máximos de rigidez das vigas; as variações dos valores de módulo de elasticidade dinâmico das lamelas foram afetadas pela amplitude dos valores de densidade e de velocidade de propagação da onda; o posicionamento correto das lamelas, por meio de métodos dinâmicos de classificação, teve como consequência o aumento do módulo de elasticidade e houve uma correlação muito baixa entre o método dinâmico e estático das vigas, não sendo possível a elaboração de equação adequada de correlação entre as diferentes formas de obtenção da variável.
\end{abstract}

Palavras-chave: Vigas estruturais de madeira, técnica de ondas de tensão, rigidez.

\section{EVALUATION OF STIFFNESS OF STRUCTURAL GLULAM BEAMS BONDED BY POLYURETHANE RESIN}

\begin{abstract}
The objective of the study was to technically evaluate the production of Glulam beams bonded by polyurethane adhesive through the determination of the static and dynamic Modulus of Elasticity. For the confection of the structural beams pieces of lumber of Pinus taeda and polyurethane resin were used. All the pieces that composed the beams were classified visually by the number of growth rings and percentage of latewood, and the presence of defects, and later the layers were classified by the dynamic modulus of elasticity. The beams were mounted and pressed, the value of the dynamic modulus of elasticity was estimated and the static was calculated with base on static test on a universal machine. Data analysis was performed using regression analysis. It was concluded that the visual classification used in the selection of pieces was insufficient to obtain the maximum values of stiffness of the beams; the variations of the values of dynamic modulus of elasticity of the layers were affected by the swing of the values of density and wave propagation speed; the correct positioning of the lamellas through dynamic classification methods had as consequence the increase of the elasticity modulus; and there was a very low correlation between the dynamic and static of the beams, being that it was not possible to elaborate an adequate correlation equation between different ways of obtaining the variable.
\end{abstract}

Key words: Structural beams of wood, stress wave method, stiffness.

\section{INTRODUÇÃO}

A demanda por produtos com maior valor agregado, como as molduras e os painéis colados lateralmente $(E G P$ - edge glued panels), é influenciada pelas atividades de construção e decoração residencial. Entre as ameaças e oportunidades desse segmento estão o ciclo de uso dos produtos, a entrada de produtos substitutos, os concorrentes diretos, os preços e a necessidade de constante evolução do parque industrial. Entre os substitutos aos PMVA estão os produtos engenheirados, como as vigas estruturais ( $I$ - joist e a Madeira Laminada Colada (MLC), o LVL (Laminated Veneer Lumber) e os painéis de OSB (Oriented Strand Board), os quais têm se mantido em constante evolução.

Natterer (1991) afirma que uma das características da MLC é a versatilidade na obtenção das mais variadas formas geométricas para elementos estruturais. As possibilidades arquitetônicas são inúmeras e dependem da indispensável colaboração dos engenheiros florestais, industriais madeireiros, civis e arquitetos. Têm-se como principais vantagens: a facilidade na construção de grandes estruturas, a partir de peças de dimensões comerciais;

\footnotetext{
${ }^{1}$ Engenheiro Florestal, Professor Dr. em Engenharia Florestal - Departamento de Engenharia Florestal - Centro de Ciências Agroveterinárias/CAV Universidade do Estado de Santa Catarina/UDESC - Av. Luiz de Camões, 2090, Bairro Conta Dinheiro - 88520-000 - Lages, SC - a2abc@cav.udesc.br ${ }^{2}$ Engenheiro Florestal, Professor Dr. em Engenharia Florestal - Centro de Ciências Florestais e da Madeira - Setor de Ciências Agrárias - Universidade Federal do Paraná/UFPR - Campus III - Av. Lothário Meissner, 632, Jardim Botânico - 80210-170 - Curitiba, PR - jmatos.ufpr@gmail.com
}

Cerne, Lavras, v. 17, n. 4, p. 593-600, out./dez. 2011 
a redução de rachaduras e outros defeitos típicos de peças maciças de madeira; a possibilidade de emprego de peças de qualidade inferior em zonas menos solicitadas, podendo-se combinar espécies distintas; a baixa relação peso/ resistência; e o bom desempenho sob a ação do fogo. Como aspecto restritivo, pode ser citar que a MLC tem custo superior ao da madeira maciça, e requer técnicas, equipamentos e mão de obra especializada em sua fabricação.

Pesquisas desenvolvidas mostram que, para os elementos estruturais em MLC, devem ser utilizadas espécies de madeira que apresentem densidade entre $0,40 \mathrm{~g} / \mathrm{cm}^{3}$ e $0,75 \mathrm{~g} / \mathrm{cm}^{3}$ (FIORELLI, 2005). Segundo a norma ASTM D 3737 (AMERICAN SOCIETY FOR TESTING MATERIALS - ASTM, 1998), a adoção de critérios para a classificação e posicionamento na viga, garante um aumento da resistência e da rigidez dos elementos estruturais. A classificação das lâminas pode ser realizada, visualmente e mecanicamente, por métodos destrutivos em corpos-de-prova e não destrutivos como o Stress Wave Method.

Recentemente, as indústrias de adesivo apresentaram um composto alternativo para a indústria de estruturas de madeira, o poliuretano (PUR), que foi desenvolvido a fim de eliminar uma gama de problemas relacionados ao uso estrutural de madeira (VILAR, 1993). O PUR na linha de cola tem como características: alta resistência mecânica à alta umidade e temperatura; o produto não agride o meio ambiente, pois é livre de solventes e exige menor consumo de água; e atende a normas internacionais de qualidade e resistência.

Neste estudo, objetivou-se avaliar tecnicamente a produção de vigas de madeira laminada colada de Pinus taeda unidas por meio de adesivo poliuretano, pela determinação do módulo de elasticidade (MOE) estático e dinâmico.

\section{MATERIAL E MÉTODOS}

As vigas foram confeccionadas na Battistella Indústria e Comércio Ltda, Lages - SC e os ensaios foram realizados no Laboratório de Tecnologia da Madeira da Universidade Federal do Paraná. Para a confecção das vigas foram utilizadas 200 peças de madeira serrada de Pinus taeda com $12 \%$ de umidade e adesivo Poliuretano (PUR - Purbond HB 22). O experimento envolveu 7 vigas de MLC com $3100 \mathrm{~mm}$ de comprimento, $122 \mathrm{~mm}$ de largura e $156 \mathrm{~mm}$ de altura, que foram compostas por 6 peças de madeira serrada com largura de $122 \mathrm{~mm}$ e espessura de $26 \mathrm{~mm}$, coladas face a face. A análise dos dados foi realizada por meio da análise da regressão linear, que tem por objetivo encontrar uma função que permita descrever e compreender a relação entre uma variável dependente e uma ou mais variáveis independentes, além de projetar uma variável em função de uma ou mais variáveis independentes. Neste estudo, a variável independente foi o MOE dinâmico obtido em ensaio não destrutivo, por meio da Técnica de Onda de Tensão (Stress Wave Timer) e a variável dependente foi o módulo de elasticidade a flexão estática.

\subsection{Processo de confecção das lamelas}

Todas as peças de madeira serrada passaram inicialmente pelo processo de pré-bitolamento em uma plaina de 4 faces, com intuito de facilitar a visualização dos defeitos. Os processos de destopo e classificação foram feitos conjuntamente, sendo que a classificação foi baseada na metodologia da empresa, evitando que as peças fossem classificadas por métodos de difícil empregabilidade nas indústrias.

A classificação da empresa foi baseada em Carreira (2003), que utilizou os métodos do Southern Pine Inspection Bureau - SPIB (1994, 1999), os quais limitam a presença de defeitos, além de agrupar as peças em grupos de densidade. Dessa forma, classificaram-se visualmente as peças, observando-se as seguintes características:

- densidade: as peças foram classificadas em Densa (D) com 6 ou mais anéis de crescimento e mais de $1 / 3$ da seção transversal contendo lenho tardio, ou 4 ou mais anéis de crescimento e mais de $1 / 2$ da seção transversal contendo lenho tardio; e Não-Densa (ND) para as demais densidades (média e baixa).

- presença de defeitos e a densidade das peças: na classe densa não foram permitidos defeitos como nó solto, nó tipo gravata, nó firme atravessando a peça em espessura, medula em ambos os lados, esmoado e rachaduras; já, para a classe não densa foram permitidos somente a presença de nó solto e medula.

- comprimento: as peças a serem utilizadas nas extremidades não poderiam ter comprimento inferior a $800 \mathrm{~mm}$ e as do miolo inferior a $300 \mathrm{~mm}$.

$\mathrm{Na}$ junção do topo das peças, foram feitas em média 5 emendas dentadas verticais tipo finger joint por lamela, as quais foram confeccionadas com dentes de $26 \mathrm{~mm}$ de comprimento, conforme recomendação da DIN-68-140 (DEUTSCHES INSTITUT FÜR NOMUNG - DIN, 1971).

Cerne, Lavras, v. 17, n. 4, p. 593-600, out./dez. 2011 


\subsection{Determinação do MOE dinâmico das lamelas por emissão de ondas acústicas}

Inicialmente, obteve-se o peso e o volume das lamelas para a determinação da densidade. O equipamento para determinação da velocidade de onda foi o Stress Wave Timer, Modelo 239A, da Metriguard, o qual é composto por dois transdutores acelerômetros dispostos sobre o material a ser medido e um relógio registrador. Foi determinado o MOE dinâmico em todas as lamelas, pela Equação 1.

$$
M O E_{d}=V^{2} \times \delta_{12 \%} \times \frac{1}{g}
$$

sendo:

$M O E d=$ módulo de elasticidade dinâmico.

$V=$ velocidade de propagação da onda.

$\delta_{12 \%}=$ densidade a $12 \%$ de umidade).

$g=$ aceleração da gravidade.

\subsection{Processo de formação das vigas}

Antes da aplicação do adesivo, as vigas foram montadas para se distribuir as lamelas quanto ao espaçamento entre as emendas, e a disposição da classe de madeira densa nas zonas de tração e compressão da viga, duas camadas inferiores e duas superiores, respectivamente. Nas duas camadas internas, próximas a linha neutra, foram utilizadas lamelas classificadas como não densas pelo método visual.

$\mathrm{O}$ equipamento para a aplicação do adesivo foi o sistema de spray e a gramatura utilizada foi de $200 \mathrm{~g} / \mathrm{m}^{2}$ em linha simples sem a mistura de catalisador. Na prensa, as vigas foram colocadas em temperatura ambiente sob pressão de 12 Bar, durante o tempo de 12 horas para a solidificação do adesivo entre as lamelas da viga.

\subsection{Estimativa do MOE dinâmico das vigas de madeira laminada colada}

O MOE dinâmico das vigas foi estimado com base nos valores individuais de cada lamela. As duas lamelas superiores e as duas inferiores foram compostas por peças classificadas como densas e as duas lamelas centrais por peças não densas, entretanto, dentro das duas classificações não houve distribuição das lamelas por rigidez. Dessa forma, denominou-se o MOE obtido por esse método de aleatório.

As lâminas externas, de acordo com Bodig e Jayne (1982), contribuem mais sobre o valor do MOE efetivo da viga que aquelas colocadas próximas à linha neutra.
Nesse caso, o posicionamento das lâminas de maior rigidez próximas a superfície tornam-se mais eficientes. Assim, fez-se também a estimativa do MOE, pela distribuição das lamelas, de acordo com a região mais solicitada durante os ensaios, quando a lamela com maior rigidez foi colocada na superfície inferior da viga, a segunda na superfície superior, posteriormente a terceira na camada 4, a quarta na camada dois e, assim, sucessivamente. Esse procedimento foi denominado MOE não aleatório.

O cálculo do MOE estático das vigas (Equação 2), foi obtido de acordo com a fórmula apresentadas por Bodig e Jayne (1982).

$$
\operatorname{MOE}_{e}=\frac{1}{I} \times \sum_{i=1}^{n} \operatorname{MOEt}^{i} \times\left(I_{O}^{i}+A^{i} \times\left(d^{i}\right)^{2}\right) \quad \text { Equação } 2
$$

sendo:

$M O E_{e}=$ Módulo de Elasticidade Efetivo.

$M O E t^{i}=$ Módulo de Elasticidade da i-ésima lâmina na direção longitudinal.

$I=$ Momento de Inércia de toda a seção da viga.

$n=$ Número Total de Lâminas.

$I_{o}{ }^{i}=$ Momento de Inércia da i-ésima lâmina em relação a sua linha neutra, baricentral.

$A^{i}=$ Área da seção transversal da i-ésima lâmina.

$d^{i}=$ Distância entre o Plano Baricentral da Viga Laminada e a i-ésima lâmina.

\subsection{Determinação do MOE e do MOR obtidos a partir do ensaio estático das vigas}

$\mathrm{Na}$ realização dos ensaios das vigas, foi utilizada como referência a ASTM D-198 (ASTM, 1997). A distância entre os pontos de apoio na máquina de ensaios foi de $2700 \mathrm{~mm}$, sendo que à distância entre o ponto de reação até o ponto de aplicação de carga foi de 900 $\mathrm{mm}$, conforme prescrito na norma, que determina para a avaliação da flexão um comprimento da zona de cisalhamento na razão entre 5:1 a 12:1, o qual corresponde à razão entre a distância entre o ponto de aplicação da carga e o ponto de reação mais próxima e a altura da viga; sendo assim, na razão de 900 para 122, obteve-se 7,4:1. Calculada as distâncias na zona de cisalhamento, determinou-se a distância entre os pontos de aplicação de carga, que vem a ser a zona de flexão que foi também de $900 \mathrm{~mm}$.

Para a medição da deformação na linha neutra da viga no decorrer do ensaio, utilizou-se um deflectômetro que foi posicionado na linha central, sendo apoiado em um suporte de alumínio conhecido como YOKE, que estava fixado em dois pontos distanciados em $700 \mathrm{~mm}$ (L1).

Cerne, Lavras, v. 17, n. 4, p. 593-600, out./dez. 2011 
A velocidade do ensaio foi de $0,6 \mathrm{~mm} /$ minuto, suficiente para romper o material em 10 minutos, não menos que 6 minutos e nem mais que 20 minutos. $\mathrm{O} \mathrm{MOE}$ da viga (Equação 3) foi calculado com base na diferença de deformação entre $40 \%$ e $10 \%$ da força máxima, adotandose como referência para cálculo desse parâmetro a EN 789 (EUROPEAN STARDARD - EN, 1995).

$$
M O E=\frac{\left(F_{40 \%}-F_{10 \%}\right) \times\left(L_{1}\right)^{2} \times L_{2}}{16 \times\left(d e f_{40 \%}-d e f_{10 \%}\right) \times I_{o}} \quad \text { Equação } 3
$$

sendo:

$M O E=$ Módulo de elasticidade.

$F_{40 \%}=$ Carga correspondente a $40 \%$ da carga máxima.

$F_{10 \%}=$ Carga correspondente a $10 \%$ da carga máxima.

$L_{1}=$ Distância entre os pontos de apoio do YOKE.

$L_{2}=$ Metade do comprimento da zona de cisalhamento.

$\operatorname{def}_{40 \%}=$ Deformação correspondente a $40 \%$ da carga máxima.

$\operatorname{def}_{10 \%}=$ Deformação correpondente a $10 \%$ da carga máxima.

$I_{o}=$ Momento de inércia baricentral $\left(\mathrm{cm}^{4}\right)$.

O MOR foi calculado (Equação 4) com base na força máxima aplicada durante o ensaio de flexão, incluindo no cálculo o momento fletor máximo no meio do corpo de prova e o módulo de resistência da seção transversal da viga.

$$
M O R=\frac{F_{M A X} \times L_{2}}{2 \times W}
$$

Equação 4

sendo:

$M O R=$ Módulo de ruptura.

$L_{2}=$ Metade do comprimento da zona de cisalhamento.

$W=$ Módulo de Resistência.

\section{RESULTADOS E DISCUSSÃO}

\subsection{Módulo de elasticidade dinâmico das lamelas}

O método não destrutivo de avaliação da rigidez da madeira pelo Stress Wave Method depende diretamente da velocidade de propagação da onda ao longo da peça e da densidade do material. Quanto maior a velocidade, maior será o valor do MOE, mantendo-se as demais variáveis inalteradas, como pode ser observado na Tabela 1, onde a lamela denominada de M2 da viga 1 e a M1 da viga 2 , apresentam $437 \mathrm{~kg} / \mathrm{m}^{3}$ de densidade, velocidades de $4.000 \mathrm{~m} / \mathrm{s}$ e $3.861 \mathrm{~m} / \mathrm{s}$ e, consequentemente, MOE diferenciados.
Tabela 1 - Valores individuais de MOE dinâmico das lamelas utilizadas nas vigas.

Table 1 - Individual values for dynamic MOE for the lamellas

\begin{tabular}{|c|c|c|c|c|}
\hline Viga & Lam & Densid. $\left(\mathrm{kg} / \mathrm{m}^{3}\right)$ & Veloc. $(\mathrm{m} / \mathrm{s})$ & $\operatorname{MOE}(\mathrm{MPa})$ \\
\hline \multirow{6}{*}{01} & SS & 488 & 3.584 & 6.277 \\
\hline & M1 & 435 & 3.866 & 6.496 \\
\hline & M2 & 437 & 4.000 & 6.987 \\
\hline & M3 & 427 & 3.774 & 6.088 \\
\hline & M4 & 415 & 3.571 & 5.298 \\
\hline & SI & 457 & 4.695 & 10.074 \\
\hline \multirow{6}{*}{02} & SS & 413 & 3.667 & 5.559 \\
\hline & M1 & 437 & 3.861 & 6.510 \\
\hline & M2 & 428 & 4.219 & 7.630 \\
\hline & M3 & 389 & 3.444 & 4.614 \\
\hline & M4 & 404 & 3.846 & 5.979 \\
\hline & SI & 491 & 4.498 & 9.946 \\
\hline \multirow{6}{*}{03} & SS & 440 & 3.436 & 5.199 \\
\hline & M1 & 425 & 3.958 & 6.665 \\
\hline & M2 & 426 & 3.906 & 6.508 \\
\hline & M3 & 406 & 3.827 & 5.947 \\
\hline & M4 & 415 & 3.974 & 6.558 \\
\hline & SI & 403 & 4.688 & 8.858 \\
\hline \multirow{6}{*}{04} & SS & 450 & 3.911 & 6.359 \\
\hline & M1 & 445 & 3.659 & 5.954 \\
\hline & M2 & 421 & 4.000 & 6.547 \\
\hline & M3 & 404 & 4.167 & 7.016 \\
\hline & M4 & 416 & 4.127 & 7.090 \\
\hline & SI & 441 & 4.000 & 7.052 \\
\hline \multirow{6}{*}{05} & SS & 450 & 4.431 & 8.835 \\
\hline & M1 & 414 & 3.984 & 6.576 \\
\hline & M2 & 453 & 4.342 & 8.538 \\
\hline & M3 & 456 & 3.866 & 6.816 \\
\hline & M4 & 465 & 3.650 & 6.196 \\
\hline & SI & 459 & 3.676 & 6.205 \\
\hline \multirow{6}{*}{06} & SS & 440 & 3.866 & 6.572 \\
\hline & M1 & 455 & 3.827 & 6.662 \\
\hline & M2 & 427 & 3.712 & 5.894 \\
\hline & M3 & 409 & 4.143 & 7.026 \\
\hline & M4 & 437 & 4.143 & 7.498 \\
\hline & SI & 521 & 4.587 & 10.966 \\
\hline
\end{tabular}
used in beams.

Continua... To be continued...

Cerne, Lavras, v. 17, n. 4, p. 593-600, out./dez. 2011 
Tabela 1 - Continua...

Tabela 1 - Continued...

\begin{tabular}{ccccc}
\hline Viga & Lam & Densid. $\left(\mathrm{kg} / \mathrm{m}^{3}\right)$ & Veloc. $(\mathrm{m} / \mathrm{s})$ & MOE $(\mathrm{MPa})$ \\
\hline \multirow{4}{*}{07} & SS & 447 & 3.425 & 5.241 \\
& M1 & 431 & 3.942 & 6.708 \\
& M2 & 437 & 3.601 & 5.664 \\
& M3 & 435 & 3.546 & 5.466 \\
& M4 & 405 & 3.759 & 5.726 \\
& SI & 493 & 3.589 & 6.344 \\
\hline
\end{tabular}

Legenda: SS: lamela da superfície superior; M1, M2, M3, M4: lamelas centrais no sentido descendente; SI: lamela da superfície inferior. Lam: Lamela, Densid: Densidade, Veloc: Velocidade.

Ao mesmo tempo, a velocidade de propagação da onda também é influenciada pela quantidade de material lenhoso a ser atravessada no material, pois, quanto maior a variável mencionada, menor é o tempo despendido desde o emissor até o receptor da onda. Sendo a velocidade, a relação entre distância e tempo, mantendo-se a primeira variável inalterada e aumentando-se a segunda, diminuise a velocidade. Mas, em contrapartida, quanto maior a densidade, maior é o MOE, tendo em vista que é uma função do quadrado da velocidade de propagação da onda e da densidade.

Dessa forma, pode-se atribuir os diferentes índices de velocidade à presença de defeitos naturais como nós, aos encaixes utilizados na emendas dentadas tipo finger joint e ao adesivo utilizado na junção.

Com relação à densidade, pode-se destacar a influência da espécie, do teor de umidade que também influencia no desempenho do adesivo, durante o seu processo de cura, da largura dos anéis de crescimento, da posição de retirada da peça ao longo do fuste, da porcentagem de lenho inicial e tardio, da diferenciação entre lenho juvenil e adulto, além de influências externas. Dessa forma, observa-se no cálculo da densidade das peças (Tabela 1) uma grande heterogeneidade de valores, variando de 389 a $521 \mathrm{~kg} / \mathrm{m}^{3}$. Essa amplitude é derivada da origem das peças, que contempla uma série de condições, destacando-se a idade das árvores, a posição de retiradas das peças e as influências externas.

$\mathrm{Na}$ Tabela 1, apresentam-se os valores do MOE dinâmico para as vigas, de acordo com o sistema de classificação da empresa, onde observa-se que as lamelas com maiores valores de rigidez não estão posicionadas nas zonas de compressão e tração da viga.
Assim, somente a classificação visual não foi suficiente, mas, apresentou-se como um método importante para uma pré-classificação no momento do destopo e da escolha das peças que são unidas nas lamelas, evitando a execução dos ensaios não destrutivos em todas as peças destopadas, passando para essa atividade depois da lamela pronta.

O sistema de classificação pelo Stress Wave é simples, prático e de resultado imediato, podendo ser utilizado em qualquer parte da empresa, seja na floresta ou na indústria, tendo em vista que é um equipamento leve e portátil, podendo determinar a velocidade de propagação tanto em árvores, quanto em peças de madeira serrada. $\mathrm{O}$ conjunto desses fatores torna o sistema atraente frente a outros métodos não destrutivos que são de grande porte, alto investimento e manutenção, contando com softwares sofisticados.

Em estudo realizado por Fagundes e Szücs (1998), nota-se que após a classificação estabeleceram-se 3 classes de vigas com MOE maiores, médios e menores, os quais foram distribuídos em 5 modelos de vigas; os resultados demonstraram que não houve diferença estatística na resistência das vigas analisadas, mas a dispersão dos valores de MOE foi pequena, variando de $7.581 \mathrm{MPa}$ a $7.630 \mathrm{MPa}$. Entretanto, no presente estudo, a dispersão dos valores de MOE dinâmico foi de 4.614 MPa a 10.966 $\mathrm{MPa}$.

Evidencia-se, portanto, a necessidade da classificação para peças estruturais, podendo-se dizer que quanto maior o número de classes, maior será a probabilidade de se aumentar a resistência da viga. Mas, deve-se atender à questão de operacionalidade nas empresas, podendo dificultar o processo, diminuindo o rendimento.

\subsection{Módulo de elasticidade dinâmico e estático das vigas}

A partir da determinação do MOE dinâmico individual das lamelas, foi estimado o MOE dinâmico da viga com base na classificação visual realizada pela empresa e, também, pela distribuição das lamelas de acordo com a qualidade de rigidez desejada. Pela Tabela 2 , observa-se que em todas as vigas com uma classificação e distribuição adequada das lamelas, houve um ganho médio de rigidez de $6,60 \%$, pela distribuição das lamelas ao longo da viga pelo Stress Wave Timer. A distribuição das lamelas independe do adesivo que está sendo utilizado, mas diretamente das dimensões e das características anatômicas das peças que estão sendo trabalhadas.

Cerne, Lavras, v. 17, n. 4, p. 593-600, out./dez. 2011 
Tabela 2 - Módulo de elasticidade e de ruptura das vigas de MLC.

Table 2-Modulus of elasticity and rupture of the glulam beams.

\begin{tabular}{|c|c|c|c|c|}
\hline \multirow[b]{2}{*}{ Viga } & \multicolumn{2}{|c|}{ MOE Dinâmico (MPa) } & \multirow{2}{*}{$\begin{array}{c}\text { MOE } \\
\text { Estático } \\
(\mathrm{MPa})\end{array}$} & \multirow{2}{*}{$\begin{array}{l}\text { MOR } \\
(\mathrm{MPa})\end{array}$} \\
\hline & Aleatório & $\begin{array}{c}\text { Não } \\
\text { Aleatório }\end{array}$ & & \\
\hline 1 & 7.524 & 7.870 & 7.194 & 25 \\
\hline 2 & 7.301 & 7.991 & 9.629 & 23 \\
\hline 3 & 6.891 & 7.362 & 7.489 & 21 \\
\hline 4 & 6.664 & 6.962 & 7.204 & 24 \\
\hline 5 & 7.232 & 8.078 & 8.811 & 28 \\
\hline 6 & 8.245 & 8.502 & 8.255 & 32 \\
\hline 7 & 5.894 & 6.267 & 6.700 & 28 \\
\hline Média & 7.107 & 7.576 & 7.897 & 26 \\
\hline Variância & $539.411,24$ & $584.069,00$ & $1.089 .518,95$ & 13,81 \\
\hline Desvio Padrão & 734,45 & 764,24 & 1043,80 & 3,72 \\
\hline Valor Máximo & 8.245 & 8.502 & 9.629 & 32 \\
\hline Valor Mínimo & 5.894 & 6.267 & 6.700 & 21 \\
\hline
\end{tabular}

*MOE Dinâmico Aleatório: pelo SWT conforme distribuição utilizada pela empresa, ou seja, sem distribuição das lamelas por rigidez dentro das duas classes de densidade

*MOE Dinâmico Não aleatório: através do SWT, conforme distribuição por MOE em cada lamela; onde a lamela com maior valor de MOE foi utilizada na superfície inferior, a segunda na superior, a terceira na lamela denominada M4, a quarta na M1 e assim sucessivamente, em função das zonas externas serem as mais solicitadas durante a aplicação da carga.

*MOE Estático: variável determinada por meio destrutivo em Máquina Universal de Ensaios.

Neste estudo, optou-se na divisão das peças em duas classes de qualidade, densa e não densa, sem ter a preocupação com os valores de resistência dentro das classes. Assim, a resistência foi reduzida quando comparada com o que se poderia ser obtido do material.

Os valores obtidos para a variável MOE estático das vigas apresentaram uma grande dispersão entre os valores encontrados, evidenciando a influência dos fatores relacionados à madeira. A dispersão dos resultados pode ser verificada, por exemplo, na viga 7 e na 2 , onde os valores foram de $6.700 \mathrm{MPa}$ e $9.629 \mathrm{MPa}$, ou seja, uma diferença de $30 \%$ entre os valores de rigidez das vigas.

Efetuando-se uma análise comparativa entre os dois métodos de obtenção do MOE, verifica-se, pela análise da variância que os resultados não apresentaram diferença estatística (Tabela 3) e que a correlação entre as variáveis foi muito baixa, pois o coeficiente de correlação encontrado foi de 0,5243 (Figura 1). Dessa forma, evidenciou-se que MOE dinâmico explica somente parte do estático, sendo o restante derivado de outras variáveis como matéria-prima, condições de confecção das vigas e procedimentos utilizados nos ensaios.

Tabela 3 - Análise da variância para MOE dinâmico e estático.

Table 3 - Analysis of variance for dynamic and static modulus of elasticity.

\begin{tabular}{|c|c|c|c|c|c|}
\hline $\begin{array}{l}\text { Fontes de } \\
\text { variação }\end{array}$ & GL & SQ & QM & $\mathrm{F}$ & $\begin{array}{c}\text { Nível de } \\
\text { significância }\end{array}$ \\
\hline Entre grupos & 1 & $2,2185.10^{6}$ & $2,1851.10^{6}$ & 2,6829 & 4,7472 \\
\hline Dentro grupos & 12 & $9,7735.10^{6}$ & $8,1446.10^{6}$ & & \\
\hline Total & 13 & $1,1958.10^{6}$ & & & \\
\hline
\end{tabular}

SQ: Soma de quadrados; GL: grau de liberdade; QM: quadrado médio; F: valor de F calculado.

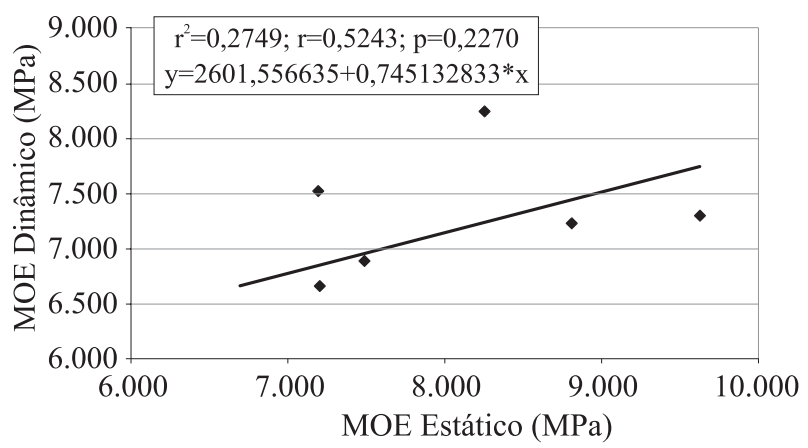

Figura 1 - Gráfico de correlação linear entre os valores de módulo de elasticidade dinâmico (estimado) e estático (real) medido em vigas laminadas coladas.

Figure 1-Graph of the linear correlation between the values of dynamic elasticity module (estimated) and static (real) measured in glulam beams.

Mesmo não havendo relação direta entre o MOE estimado da viga e o real, reforça-se a importância dos ensaios não destrutivos, pois classificando a madeira em grupos de resistência, privilegia-se o aumento da resistência das vigas durante a sua utilização na estrutura e/ou durante os ensaios mecânicos em laboratório, assegurando um melhor aproveitamento da madeira e a maior segurança das vigas em uso.

O valor do módulo de ruptura é apenas comparativo, não sendo influenciado pela relação vão e altura da viga. A análise dos valores do MOR está apresentada na Tabela 2, onde observa-se que a média foi de $26 \mathrm{MPa}$ e a variância de 13,81 . Salienta-se que as rupturas nas vigas ensaiadas foram observadas nos estágio finais da deformação 
plástica, sem grandes variações na proporção de deflexão, em relação ao limite proporcional.

Andrigheto e Szücs (2006) realizaram ensaios de flexão estática em MLC de Pinus spp com $3150 \mathrm{~mm}$ de comprimento e $315 \mathrm{~mm}$ de altura, composta por 12 lâminas classificadas de acordo com análise visual e propriedades físicas / mecânicas da madeira. Os resultados evidenciaram valores médios de MOE de $12.695 \mathrm{MPa}$ nas vigas com $1 / 4$ de lâminas com qualidade superior; para as vigas com $1 / 6$ de lâminas com qualidade superior, os valores de MOE foram de $10.252 \mathrm{MPa}$; já, quando foram utilizadas lâminas invertidas, ou seja, lâminas no sentido vertical nas regiões de maior esforço, o MOE foi de $11.685 \mathrm{MPa}$.

Em estudo realizado por Nielsen (1998) com MLC de Eucalyptus grandis, com seção retangular de $10 \mathrm{X}$ $23 \mathrm{~cm}$, formadas com adesivo Resorcinol-Formaldeído e com peças de árvores com idade média de 9 anos, de duas procedências distintas, foram encontrados valores de MOE de $10.885 \mathrm{MPa}$ para densidade média de $630 \mathrm{~kg} / \mathrm{m}^{3}$; e 11.607 MPa para densidade média de $680 \mathrm{~kg} / \mathrm{m}^{3}$.

Comparando-se os resultados, verifica-se que os valores de MOE em MLC, tanto de Pinus spp como de Eucalyptus spp, foram superiores ao maior valor encontrado no presente estudo, que foi de 9.629 Mpa. Pode-se dizer que as vigas apresentaram maior deformabilidade e menores índices de rigidez e resistência. Deve-se enfatizar que os valores de MOE encontrados para as vigas de MLC podem ser aumentados com o efetivo controle dos níveis de defeitos da madeira admitida para a classificação.

\section{CONCLUSÕES}

O sistema de classificação visual utilizado na seleção de peças para a confecção de vigas foi insuficiente para se atingir os valores máximos de $\mathrm{MOE}$ das vigas. As variações dos valores de MOE dinâmico das lamelas foram afetadas diretamente pela amplitude dos valores de massa específica e de velocidade de propagação da onda. $O$ posicionamento correto das lamelas, pelo método dinâmico de classificação, teve como consequência o aumento do módulo de elasticidade. Não houve correlação entre o módulo de elasticidade dinâmico e estático das vigas, não sendo possível a elaboração de equação adequada de correlação entre as diferentes formas de obtenção da variável.

\section{REFERÊNCIAS}

AMERICAN SOCIETY FOR TESTING MATERIALS.

ASTM D-198: standard test methods of static tests of lumber in structural sizes. Philadelphia, 1997. 26 p.
AMERICAN SOCIETY FOR TESTING MATERIALS. ASTM

D-3737: standard practice for establhishing stresses for structural glued laminated timber. West Conshohoken, 1998. 28 p.

ANDRIGHETO, R.; SZÜCS, C. A. Estudo comparativo entre madeira laminada colada e madeira laminada colada multicolada. In: ENCONTRO BRASILEIRO EM MADEIRAS E EM ESTRUTURAS DE MADEIRA, 10., 2006, São Pedro. Anais... São Pedro: EBRAMEM, 2006. CD-ROM.

BODIG, J.; JAYNE, B. A. Mechanics of wood and wood composities. New York: V. N. Reinhold, 1982. 736 p.

CARREIRA, M. R. Critérios para classificação visual de peças estruturais de Pinus sp. 2003. 182 p. Dissertação (Mestrado em Engenharia de Estruturas) - Universidade de São Paulo, São Carlos, 2003.

DEUTSCHES INSTITUT FÜR NOMUNG. DIN 68 140: wood finger, jointing. Berlin, 1971.

EUROPEAN STARDARD. EN 789: estruturas de madeira: métodos de teste: determinação das propriedades mecânicas de painéis derivados de madeira. Bruxelas, 1995.

FAGUNDES, G. S.; SCÜCS, C. A. Composição racional de vigas de madeira laminada colada de pinus. In: ENCONTRO BRASILEIRO EM MADEIRAS E EM ESTRUTURAS DE MADEIRA, 6., 1998, Florianópolis. Anais... Florianópolis: EBRAMEM, 1998. p. 277-288.

FIORELLI, J. Estudo teórico e experimental de vigas de madeira laminada coladas reforçadas com fibra de vidro. 2005. 108 p. Tese (Doutorado em Ciência e Engenharia de Materiais) - Universidade de São Paulo, São Carlos, 2005.

NATTERER, J. Quality criteria for timber design. In: INTERNATIONAL TIMBER ENGINEERING CONFERENCE, 2., 1991, London. Proceedings... London, 1991. p. 19-26.

NIELSEN, I. R. Utilização da madeira comercial do híbrido de Eucalyptus urophylla S.T. Blake X Eucalyptus grandis Hill ex Maiden na confecção de vigas laminadas coladas. 1998. 109 p. Dissertação (Mestrado em Engenharia Florestal) Universidade Federal do Paraná, Curitiba, 1998.

SOUTHERN PINE INSPECTION BUREAU. Graders manual for boards and 2 dimension. Pensacola, 1999.

Cerne, Lavras, v. 17, n. 4, p. 593-600, out./dez. 2011 
SOUTHERN PINE INSPECTION BUREAU. Standard grading rules for southern pine lumber. Pensacola, 1994.
VILAR, W. D. Química e tecnologia dos poliretanos. São Paulo: Grupo Pronor, 1993.

Cerne, Lavras, v. 17, n. 4, p. 593-600, out./dez. 2011 